\title{
THE PATH TO TRANSFORMATION, NAVIGATING THE BARRIERS TO FORMING TRANSIENT AND TRANSITIONAL LEARNING GROUPS IN INTERPROFESSIONAL EDUCATION
}

\author{
D. Gurbutt, P. Milne. \\ University of Central Lancashire (UNITED KINGDOM)
}

\begin{abstract}
The creation of a new medical school provided new opportunities to develop interprofessional education (IPE) with a pedagogic basis in transformational learning. The programme involved some transient groups - medical students working with different professional groups throughout the year and some learning interventions which proved to be transitional for students in intended and sometimes unintended ways. The path to transformational learning in interprofessional education requires the navigation of different perspectives and beliefs about learning, an element of tenacity and bravery in relation to holding to core pedagogic beliefs and an openness to co-production and evolution of learning elements.

Some learning episodes formed a transformative space within a particular time bounded location whilst others led to ongoing relationships and social connections beyond the classroom. Feedback indicated that transformation is not always comfortable or linear for students and the programme identified areas of challenge for both learners and staff as well as opportunities for development and co-creation.

This was a large IPE initiative involving staff and students from 9 disciplines across 7 schools and 3 faculties with involvement from service users and stakeholders. The programme incorporated almost 2000 separate student learning episodes. This paper will consider the programme from the perspective of students, creators of the learning elements and participatory staff from different disciplines, exploring the challenges, gains and learning from the initiative.

In considering these we suggest that social learning paradigms can help to give insight into their solution. For example, the intended and unintended outcomes could be considered through the perspectives of an integrated social learning paradigm. From the empirical data it is suggested that the paradigms of Activity theory and Complex adaptive systems theory give insight to how intended and unintended transformative learning takes place. These are inter-related as shown in the diagram.
\end{abstract}

Using complex adaptive system theory as part of the activity system theory paradigm, the interrelationship between the components of the suggested social learning paradigm may be viewed as complex with any one component able to take precedent over the other.

It is suggested that the unintended transformative learning and the social connections established outside the classroom arose as a result of the mixed student professionals' classroom moving through a process of having become a 'self-organising system'. The students have spontaneously produced a system that could not have been predicted from 'a knowledge of the previous state' (i.e. the facilitated teaching session).

Analysis of the empirical data through the lens of social learning theories gives insight into the "how and why' transformative learning takes place and might help to plan IPE sessions with greater confidence.

Keywords: Medical Education; Interprofessional Education; curriculum design

\section{INTRODUCTION}

In 2015, the University of Central Lancashire (UCLAN) became one of the first post -1992 universities in the UK to be given approval by the General Medical Council (GMC) to deliver, medical undergraduate education, the MBBS, in the UK as part of a newly created medical school. In the September of that year, 36 International Students from around the globe joined the programme. This was a unique venture as all students on the programme in the first year were international students with differing insights into the UK Health care systems and the National Health Service (NHS).

Opening a new medical school provided a rare opportunity to develop a whole curriculum from a 'blank sheet.' Clearly the programme had to conform to the requirements of the professional statutory body for Medicine (GMC) and in particular the guidelines provided in, initially, 'Tomorrows Doctors' (1993) [1] 
and then, the 'Outcomes for Graduates' document (2015) [2] , but there was also the freedom to shape a new curriculum based on the pedagogic values and clinical educational experiences of the core curriculum team. This provided opportunities and challenges for the team developing the programme. The clinical writing team were keen to involve service users in the design, delivery and evaluation of the programme, keeping a clear focus on patient outcomes. This driver emerged from the experiences of the practitioners and educators themselves, but also the reports of the enquiry into failings in the MidStaffordshire hospital trust (Francis, 2013) [3] and the resultant emphasis on the importance of 'values' in health and social care programmes (NMC 2014, GMC 2015.) [4]. A further element was the emergence of 'Integrated Care' within the UK as a driver for moving practice forward (NHS, 2014) [5], which in turn led to an emphasis on interprofessional education. The team were guided by the work of the Centre for Advancement of Interprofessional Education, (CAIPE) [6] and in particular their definition of Interprofessional Education (IPE) also referred to within Medicine, as Interprofessional Learning (IPL), in which meaningful learning from and between learners is emphasised along with the need for IPE to ultimately provide a positive impact on patient outcomes. A further pedagogic driver was the consideration of the ways in which learners learn both within structured learning event and more informal settings and the importance of context.

Owing to the development of not only a 'new' curriculum, but the adoption of particular pedagogy and values underpinning its development, the team gained ethical approval to conduct research not only with the medical students themselves, but also with the cohorts working alongside the MBBS students and the staff groups involved in curriculum delivery for the whole of the learning journey of the first cohort to enter the programme. Each session was evaluated using questionnaires and the overall programme was evaluated using some small focus groups. This paper considers data from the first two years of the programme as the first cohort have yet to progress through the whole course.

\section{CONTEXT}

The Teaching Excellence Framework (GovUK, 2017) [7] which was recently introduced in the UK places a strong emphasis on opportunities for students to gain employability skills and also to engage in Interdisciplinary work. The Research Excellence Framework (HEFCE, 2017) [8] also encourages interdisciplinary activity in terms of research. The UCLan Learning and Teaching Strategy (UCLan, 2017) [9] highlights the importance of: strong pedagogy, creative curriculum alongside employability and enterprise for our students. This context of documents encouraging IDE alongside the professional body requirements created a fertile ground for the creation of an innovative new programme for MBBS students which would enable them to work with professions engaged in Health and Social Care, but also in sessions with wider disciplines. In all there were 11 episodes of interdisciplinary learning over the course of the IPE programme, involving students from three faculties and seven Schools. The collaborative learning included students from a range of nine disciplines including: Medicine, Pharmacy, different Nursing programmes and specialisms, Medical Sciences and Paramedic practice and some sessions included wider discipline areas too, such as creative design. 1344 evaluations were initially analysed from the parts of the programme which focused on health and social care interprofessional education. The curriculum development team wanted to provide meaningful interprofesssional education for all participants in line with the CAIPE definition providing not just shared learning but participative sessions where students are actively interacting with each other rather than just being taught together. This provided a number of challenges. The pedagogic approach had been identified, based on the earlier work of Milne (2007) [10] in using Activity Theory (Engestrom et al, 1995) [11] and the experiences of Gurbutt (Gurbutt and Gurbutt, 2015) [12] in using creative educational approaches the decision was made to focus on transformational learning paying particular attention to the spaces and places in which students learn, understanding that not all learning is formal and that creating spaces for informal learning matters. There was also a shared interest in addressing the particular learning needs of the cohorts involved.

\section{OPPORTUNITIES TO LEARN}

This part of the paper concerns itself with the application of social learning paradigms that help to give initial insight into the 'how and why' of the IPL programme evaluation data. At the core of this evaluation is 'what sort of learning?' takes place during these sessions.

Its focus is to consider the role of complexity theory (complex adaptive systems) and social learning theories. In particular, the interrelationship of the role of Activity theory and complexity theory. In terms of complexity terminology and for the purposes of this paper learning is defined as 
"The overall thought process by which a system's emergent properties are transformed into adaptive innovations" (Bleakley 2010) [13].

This is to distinguish from informed learning where information is given to students.

Cognitive theory has held sway in the development of medical and health education in recent decades with its focus on the development of the individual doctor both at undergraduate and postgraduate training. Using cognitive theory, context is often side-lined or ignored so that overall it feels as if only part of the learning experience is represented. In part this arises out of the continuing culture of 'heroic individualism, autocracy and meritocracy' (Bleakley 2010) [13] which predominate in medicine despite the changes within UK National Health Service which advocate team working and interprofessional (IPL) collaboration.

If we are to understand the 'how and why' of the evaluation data in this paper IPL then the array of social learning paradigms can help to give insight. These would include activity theory, actor network theory and complex adaptive system theory.

The IPL sessions were based on short scenarios within various imagined contexts were played out by professional actors. Each scenario had flaws in its interprofessional communications and with the 'patients'. Small groups of mixed professional groups followed the scenarios. These were facilitated by professionals from healthcare backgrounds. If we accept that complexity theory acts as an 'informing framework' (Bleakley 2010) [13] then the social learning paradigms can be imagined as sub systems that give more insight to the data and possibly explain some of the evaluation data.

Activity systems with their components of rules and regulations, community of practice and division of labour and their complex interaction with artefacts or common objects give some insight into the data. Each participant in the IPL sessions can be said to have an activity system and the interactions between each component is complex [see Fig.1]. The complex interactions between the components of an individual's activity system with another result in outcomes in which new learning is produced.

During each session or scenario, the students' assembled in a lecture theatre and watched the scenario with 'forum theatre facilitation'. Following the initial hour of the 2-hour session the large student group was dispersed in mixed professional groups to small 'break out' rooms. At this point it is suggested that the complex interplay within individuals' activity systems is influenced by the element of 'space and place' and their meaning as artefacts. Although both spaces could be conceived as neutral, a lecture theatre is where information is received, a small discussion room enhances conversation and has the potential for collaboration. For the students it could be argued that the two spaces reify different culturohistorical perspectives. The evaluation data reflects the resultant collaboration and the reported subsequent socialisation following the end of the session illustrate the transformation that took place as a result of the interaction between the individual activity systems. In terms of complexity the groups become self-organising systems.

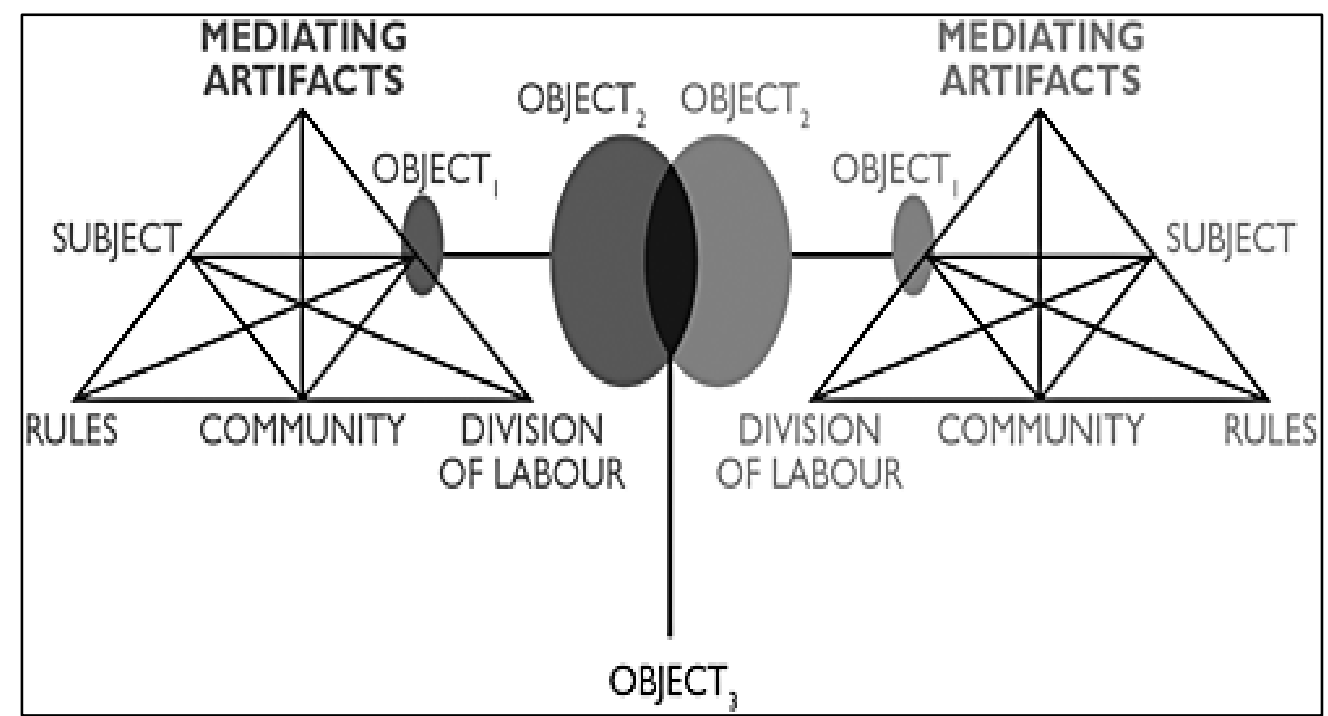

Figure 1. Activity theory as a sub system of complexity From: Engeström, Y. (1987). Learning by expanding: An activity-theoretical approach to developmental research. Helsinki: Orienta-Konsultit.[14] 
In the scenarios played out in the IPL sessions the 'subjects' are the mixed health professional groups, objects $1 \& 2$ the scenario, and the mediating artefacts interprofessional collaboration. The resultant complex interaction results in interprofessional collaboration. Object 3 can be characterised is the result of transformation.

However, this assumes a level trajectory for the complex system. As mentioned the evaluation, data noted discord on a small number of occasions in the small groups. An illustration of this was the claim by one set of students that they "did all the work". Activity theory would suggest a disjunction in the area of 'division of labour'

'Complexity as framework' labels these events as 'perturbations' which ultimately deflect the trajectory and in that moment produces different learning. These are inevitable in any complex organisation. The above example would suggest that social learning paradigms have the potential to understand the cause of perturbations.

\section{EVALUATION DATA}

Evaluations were conducted with all participants following each session and this resulted in early analysis of 1344 evaluations from Health and Social Care students participating in the 'forum theatre' scenario based sessions of the course. These questionnaires could be evaluated by: Cohort, Event, Discipline and Subject. Particular themes emerged from the findings. $83 \%$ of students felt that working interprofessionally had been beneficial and $88 \%$ felt that it had impacted their practice already, or would impact their practice. Comments included: 'I have learned so much about the work of other people' and 'these sessions have given me insight into the roles of other people.'

\subsection{Learning}

\subsubsection{Learning - Spaces and places}

Spaces and places are as important as subject content and are as much discovered as created which was evident in some of the comments about learning in a particular place or a particular way:

'I quite like the breakout discussions and I find them useful. But it does depend on who is in the room and who is leading the discussion.'

'the actor-led sessions are good, but I find that I can be quite passive - I don't really do too much in the small group discussions within the main session'

'A whole day is too much, but when we just have a morning, that works well.'

'it depends on how I feel, sometimes I really enjoy it and other times I am just thinking about all the assessments I need to complete. It can all be time consuming.'

There were also a minority of students who found that the approach did not work for them, or who maybe have a difficulty in using modes of learning other than those which form the core of their own programme of study, comments included:

'I don't like IPE, I don't like working with other professional groups.....'

'I would rather just be told stuff than have to do all of this groupwork.'

'There is no exam, so I don't feel it is very important compared with all the other things we do.'

These comments reflect a need to maybe explain more fully the benefits of working with other professional groups in this way and also to emphasise to students that learning serves a useful function beyond success in examinations. It could be argued that IPE required students to stretch into unfamiliar territory which some found challenging. This was reflected in comments such as:

'I don't know why we have to keep mixing up, why can't I stay with friends?'

'I am not good with strangers'

'we do this (communication skills) in other places, so why do we have to do it here?

'I would rather work with people I know.'

Although students might find these settings challenging, it will be interesting to see whether exposure to these challenges with the safe space of the curriculum make their interactions with unfamiliar people 
easier in practice and whether an evaluation later in the programme may stimulate reflection on this learning from a different perspective.

\subsubsection{Learning - emergent Identity}

Other qualitative data demonstrated the importance students placed on their own sense of emergent identity as practitioners:

'The sessions where I felt that I knew something that I could confidently share felt really good.'

'When the discussion was on my territory I felt fantastic.'

'The nurses knew so much, I envied them their easy confidence in talking about practice'. (The nursing students in this instance had had a longer period in placements than the medical students)

'Sometimes I felt that there was so much I still had to learn...'

'When the service-users were facilitating sessions and sharing experiences, I think "this is why I came. This is what I am here for."

Other comments included reflections on learning from others:

'I expected to learn about their work, but I learned more about how they make decisions and picked up some tips on how to negotiate.'

'What you learn isn't what you think you are going to learn. I have learned about myself and some things are surprising.'

'I often think: 'I have learned something about myself and others that I didn't know before I came."

These comments are just a small selection of the qualitative feedback, but do indicate the multiple ways in which students are learning in IPE, from content to context, about others and about themselves. Whilst the majority of students wholeheartedly embrace the opportunity to engage in IPE, for others this area of the curriculum, which is not specifically assessed by examination, is not valued to the same extent as examinable areas. Others may not recognise the 'value-added' element of the IPE until much later, suggesting that evaluations which take place at the end of the course may provide some useful triangulation on data which is generated in the earlier part of the programme.

\section{CONCLUSIONS}

Challenges persist in relation to designing and delivering IPE programmes. There may be a tension between what students 'want 'and what their educators feel that they 'need' for professional practice; for example, building confidence in communication with other practitioners may not be seen as important as other areas of study pre-placement. Students may value examinable components of the course more than non-examinable elements and the culture becomes important in promoting, enabling and valuing IPE. It is apparent that different disciplines may view themselves and their learning in different ways and IPE provides a space in which not only students, but also teaching staff learn from and between each other about how and where learning takes place and how this knowledge can inform curriculum design for integrated care.

Medicine continues to have a clinical culture which is based on a "discourse of the heroic individual and a cult of personality and uncritical modelling" and where "knowledge is treated as private capital" (Bleakley 2010) [13]. Complexity theory and its social learning paradigm sub systems offer the opportunity to innovate in medical education. CAIPE defines IPE as "Occasions when two or more professions learn with, from and about each other to improve collaboration and the quality of care". Inherently this acknowledges and implicitly requires the increased use of complexity and its social learning paradigms in medical and health professional education. Use as many sections/subsections as you need.

\section{REFERENCES}

[1] GMC, Tomorrow's Doctors - Outcomes and Standards for Undergraduate Medical Education'. 2009. Accessed 141 2018. Retrieved from: https://www.gmcuk.org/Tomorrow_s_Doctors_1214.pdf_48905759.pdf. 
[2] GMC, Outcomes for Graduates, 2015. Accessed 1412 2018. Retrieved from: https://www.gmcuk.org/Outcomes_for_graduates_Jul_15_1216.pdf_61408029.pdf.

[3] R. Francis, Report of the Mid-Staffordshire NHS Foundation Trust Public Inquiry, 2013. London: Stationary Office. Retrieved from: http://www.midstaffspublicinquiry.com/report. Accessed 141 2018.

[4] NMC, Standards for Competence for Registered Nurses, 2014. Accessed 1412 2018, Retrieved from: https://www.nmc.org.uk/standards/additional-standards/standards-for-competence-forregistered-nurses/

[5] NHS, Five Year Forward View, 2014 Accessed 1412018. Retrieved from:https://www.england.nhs.uk/wp-content/uploads/2014/10/5yfv-web.pdf

[6] Centre for Advancement of Interprofessional Education, Accessed 141 2018. www.caipe.org/

[7] GovUK, Teaching Excellence Framework, 2017, Accessed 141 2018. Retrieved from: https://www.gov.uk/government/news/universities-rated-in-teaching-excellence-framework Dept of Education.

[8] HEFCE, Research Excellence Framework, 2017. Accessed 14 12018. Retrieved from: http://www.ref.ac.uk/ London: HEFCE

[9] University of Central Lancashire. Learning and Teaching Strategy: Student Access, Experience and Success. 2017. Preston: UCLan.

[10] P. Milne, Contesting the Freedom to Learn: culture and learning in a British general practice. PhD thesis, UCLan, 2007.

[11] Y. Engeström,..,R. Engeström,M. Karkkainen, Polycontextuality and Boundary Crossing in Expert Cognition: Learning and Problem Solving in Complex Work Activities. Learning and Instruction, Vol.5, pp. 319-336. 1995.

[12] D.J. Gurbutt, R. Gurbutt (2015) 'Telling Tales - creating a space for stories in practitioner education.' In 'Creative Education, Teaching and Learning: Creativity, engagement and the student experience. Brewer G. and Hogarth, R. (Eds), London: Palgrave Macmillan

[13] A. Bleakley. Blunting Occam's razor: aligning medical education with studies of complexity. Journal of Evaluation in Clinical Practice. Vol. 16, 4, pp.849-855, 2010.

[14] Y. Engeström, Learning by expanding: An activity-theoretical approach to developmental research. Helsinki: Orienta-Konsultit, 1987. 